\title{
Mineração
}

\section{Microestruturas de pelotas feitas com mistura de redutor e finos de minério}

\author{
Jader Martins \\ Professor Titular, Depart. de Eng. de Minas, Escola de Minas, Universidade Federal de Ouro Preto, MG \\ E-mail: jadermar@zaz.com.br \\ Cesar Mendonça Ferreira \\ Professor Adjunto, Depart. de Eng. Geológica, Escola de Minas, Universidade Federal de Ouro Preto, MG \\ E-mail: cesar@feop.com.br \\ Neymayer Pereira da Silva \\ Engenheiro de Minas - SAMITRI Mineração SA \\ E-mail:neymar.lima@cvrd.com.br
}

\section{Resumo}

Na última década, especial atenção tem sido dada para os novos processos de produção de ferro primário, que, além de aproveitar matérias-primas que não podem ser utilizadas pelos processos convencionais, não apresentam os graves problemas ambientais que ocorrem principalmente nas siderúrgicas integradas. O Processo de Redução Direta a Partir de Pelota Crua (RDPC) já descrito em outros trabalhos, está incluído no rol daqueles processos em que os problemas ambientais que ocorrem nas coquerias, sinterizações e unidades de pelotização convencional, praticamente não existem.

Além disso, apresenta outras vantagens sobre os processos convencionais, particularmente o de utilizar concentrados de minério de ferro, provenientes de usinas de concentração por flotação, sem que seja necessário moer o minério e a fonte redutora até a granulação fina que se faz necessária no processo convencional de pelotização.

Esse trabalho visa a apresentar algumas características das microestruturas de pelotas feitas com a mistura de finos de agente redutor e finos de minério depois de serem submetidas a um processo de aquecimento em várias condições de tempo e temperatura.

Palavras-chaves: Pelota, redução, microestrutura.

\section{Abstract}

New processes utilised for the production of ferro primario has been receiving special attention over the last 10 years. This is due to the necessity to use high grade concentrates which can not be utilised straightforward by the steel mills and do not cause the environmetal problems produced by traditional process. The Process of Direct Reduction from Green Pellets (RDPC) reported in other works is one of those processes in which the problens associated to coking, sinterization and pelletization do not happen.

Besides, this new process has other advantages such as the possibility to use the flotation concentrates without the necessity to grind it to the same level as it occurs with the conventional pelletization.

This work aims to show some characteristics of the microstructures of pellets made out from the of mixture reducing agent and iron ore fines when they are heated under various conditions of time and temperature.

Keywords: Pellets, reduction, microstructure.

Artigo recebido em 03/06/2002 e aprovado em 12/07/2002. 


\section{Introdução}

A indústria siderúrgica tem como principal objetivo a fabricação de gusa e aço. No Brasil, isto tem sido realizado através de siderúrgicas integradas, que utilizam o coque como elemento redutor, como é o caso da USIMINAS, AÇOMINAS, CSN e COSIPA. O Brasil se destacou também no cenário mundial da indústria siderúrgica, com a utilização de biomassa (carvão vegetal) e minério de ferro bitolado (pellet ore) em pequenos altos-fornos. Os chamados guseiros se estabeleceram no Estado de Minas Gerais devido a abundância de matériasprimas, tais como: carvão vegetal, minério de ferro bitolado e calcário.

Na última década, especial atenção tem sido dada para os novos processos de produção de ferro primário, que, além de aproveitar matérias-primas que não podem ser utilizadas pelos processos convencionais, não apresentam os graves problemas ambientais. O Processo de Redução Direta a Partir de Pelota Crua (RDPC), já descrito em outros trabalhos, está incluído no rol daqueles processos em que o problema ambiental que ocorre nas coquerias, sinterizações e unidades de pelotização convencional não existe. Além disto, pode apresentar uma série de outras vantagens sobre os processos convencionais, particularmente o de utilizar concentrados de minério de ferro, provenientes de usinas de concentração por flotação, sem que seja necessário moer o minério e a fonte redutora até a granulação fina que se faz necessária no processo convencional de pelotização. Somente essa etapa representa aproximadamente $20 \%$ do custo operacional de produção de uma usina de pelotização. É importante salientar que o processo convencional de pelotização, que recentemente passou por um "boom" devido à demanda por matériasprimas de alta qualidade exigidas pelos processos de redução direta, agrega muito pouco valor ao produto, quando comparado ao ferro-esponja. Enquanto a tonelada de pelota é vendida por aproximadamente 35 dólares, a tonelada de ferro-esponja tem um preço de venda de 130 dólares.

Esse trabalho visa a apresentar algumas características das microestrutu- ras de pelotas feitas com a mistura de finos de agente redutor e finos de minério após serem submetidas a um processo de aquecimento em várias condições de tempo e temperatura em um forno-túnel contínuo de laboratório.

\section{Materiais e métodos}

Uma descrição completa dos materiais se encontra em outro trabalho apresentado por Martins et all (1999).

É importante salientar que o minério de ferro utilizado possuía teor de $97,45 \% \mathrm{Fe}_{2} \mathrm{O}_{3}$ e $2,34 \%$ de $\mathrm{SiO}_{2}$, com granulação de $100 \%$ abaixo de $100 \mu$ m e $33 \%$ menor que $35 \mu \mathrm{m}$.

Os redutores utilizados possuíam as características apresentadas nas Tabelas 1 e 2 .

\section{Forno-Túnel Contínuo}

O forno-túnel contínuo utilizado possui seção trapezoidal com largura de 95x100 mm e altura de 100 mm e três módulos de $90 \mathrm{~cm}$ de comprimento: o primeiro para aquecimento, o segundo para temperatura máxima e um terceiro módulo, sem os elementos resistíveis, atuou como zona de resfriamento.

O tempo de residência em cada módulo, quando a vagoneta operava à velocidade máxima de $6 \mathrm{~cm} /$ minuto, foi de 15 minutos.
Estabeleceu-se uma temperatura de trabalho de $1100^{\circ} \mathrm{C}$, uma vez que a temperatura máxima do forno era de $1200^{\circ} \mathrm{C}$.

Alguns testes foram realizados no forno mufla, utilizando-se de cadinhos de porcelana. Como o forno era limitado na sua potência (capacidade de aquecimento), duas taxas de aquecimento foram utilizadas: aquecimento lento $\left(15^{\circ} \mathrm{C} /\right.$ minuto) e aquecimento rápido (colocando o cadinho à temperatura máxima).

Após conhecer melhor o comportamento das pelotas com a realização dos testes em forno mufla, deu-se início aos testes em forno túnel contínuo. Depois da realização de uma série de testes no forno contínuo, percebeu-se que as pelotas dentro das vagonetas estavam bastante heterogêneas. A camada superior sofria redução, enquanto a camada inferior, devido a um processo de impermeabilização do leito, não sofria grandes transformações devido a falta de contato entre as pelotas e a atmosfera do forno. Decidiu-se por aumentar a "oxigenação" do leito, fazendo um elevado número de perfurações nas paredes laterais das vagonetas. Ainda assim, o problema não foi resolvido. Persistiu a falta de homogeneidade no leito. A melhor solução encontrada foi manufaturar uma cesta com malha de aproximadamente 5 mm de abertura, que, por ter dimensões inferiores às da vagoneta, ao ser colocada em um suporte dentro da mesma, permitiu que todos os lados do recipiente,

Tabela 1 - Análise química de carvão e coque.

\begin{tabular}{c|c|c}
\hline \multirow{2}{*}{ Elemento Químico } & \multicolumn{2}{|c}{ Teor (\%) } \\
\cline { 2 - 3 } & Carvão & Coque \\
\hline Carbono & 55,85 & 90 \\
\hline Voláteis & 35,48 & 8 \\
\hline Cinza & 2,41 & 0,15 \\
\hline Umidade & 6,26 & 1,85 \\
\hline Total & 100 & 100 \\
\hline
\end{tabular}

Tabela 2 - Reatividade de carvão e coque (norma ABNT NBR 10513).

\begin{tabular}{c|c|c|c}
\hline Material & Ensaio 1 & Ensaio2 & Média \\
\hline Coque & 17,98 & 19,28 & 18,63 \\
\hline Carvão & 99,6 & 98,85 & 99,23 \\
\hline
\end{tabular}


inclusive o fundo, tivessem um amplo contato com a atmosfera do forno.

De acordo com a composição química do minério, a quantidade estequiométrica de cada redutor, necessária para que a redução ocorra de maneira completa, foi para carvão e coque, respectivamente, 26,2 e $16,3 \%$, em relação ao minério.

\section{Resultados e discussão}

O objetivo principal desse estudo da microestrutura das pelotas reduzidas foi buscar entender alguns mecanismos do processo de redução de pelotas. Como se trata de um processo em que o redutor está intimamente misturado no estado sólido com o minério, esse processo apresenta características bastante distintas dos processos de redução convencionais (alto-forno e redução direta) e também do processo de redução em leito fluidizado. Certamente, os mecanismos que ocorrem no processo RDPC devem ser bem mais complexos do que aqueles já conhecidos das reações tipo topoquímica que ocorrem no alto-forno.

É provável que as reações topoquímicas ocorram simultaneamente tanto na parte externa da pelota como no interior da mesma à nível do grão. Não foi objetivo desse trabalho estudar exaustivamente as etapas que ocorrem durante o processo de redução, mas procurar fazer uma associação de alguns aspectos da microestrutura com aqueles resultados obtidos nos estudos das variáveis de processo.

Na Foto 1, são mostrados os efeitos da quantidade de agente redutor em testes feitos com coque, cujo grau de metalização foi de 4,8\%. É interessante observar que as regiões metalizadas se encontram na parte externa das partículas e também distribuídas de maneira homogênea por toda pelota.

Na Foto 2, onde houve um grau de metalização de $43,3 \%$, é possível observar que a distribuição do metal também ocorre de maneira homogênea. Algumas partículas estão completamente metalizadas e outras estão parcialmente.

$\mathrm{Na}$ Foto 3, onde se obteve um grau de metalização de $89,3 \%$, é possível observar a homogeneidade da redução, na qual, em nível microscópico, como era de se esperar, as reações devem ocorrer de forma topoquímica, uma vez que os óxidos se encontram na região central dos grãos.

Na série de fotos citadas anterior-

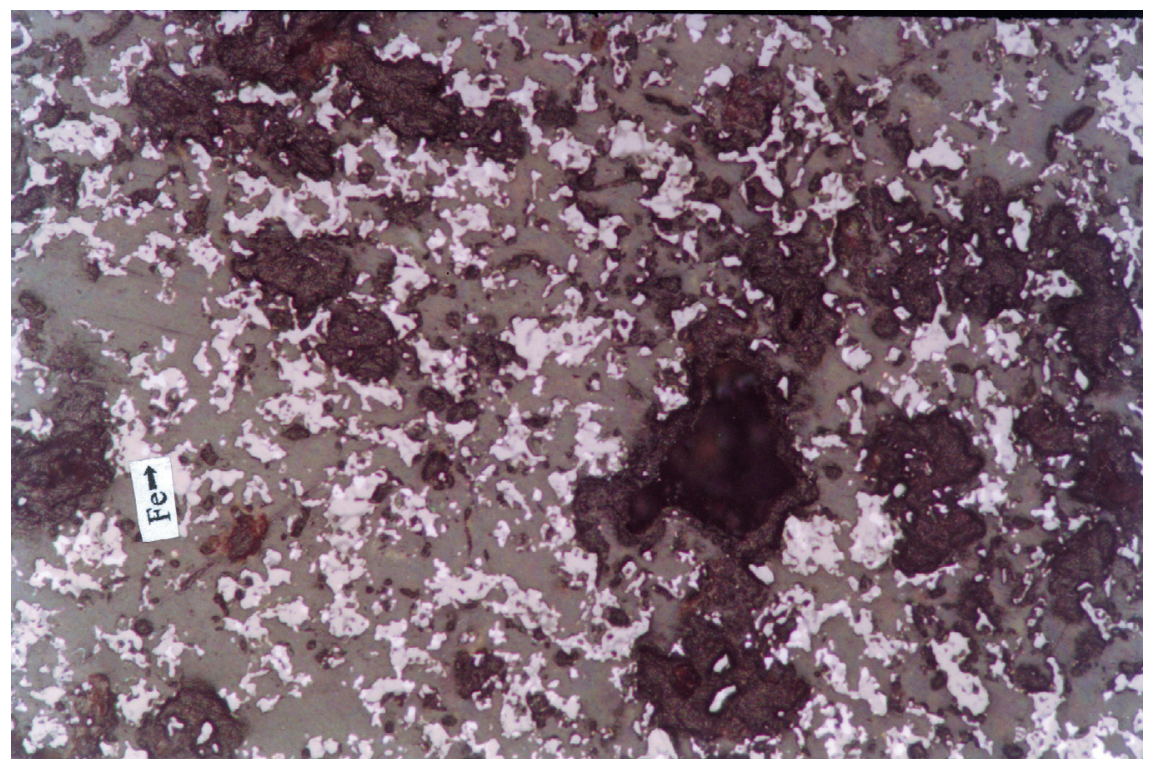

Foto 1 - Teste 13 realizado com coque $\left(12,2 \%\right.$ em peso) à temperatura de $1120^{\circ} \mathrm{C}$, tempo de 30 minutos e com 4,8\% de metalização (centro 100x).

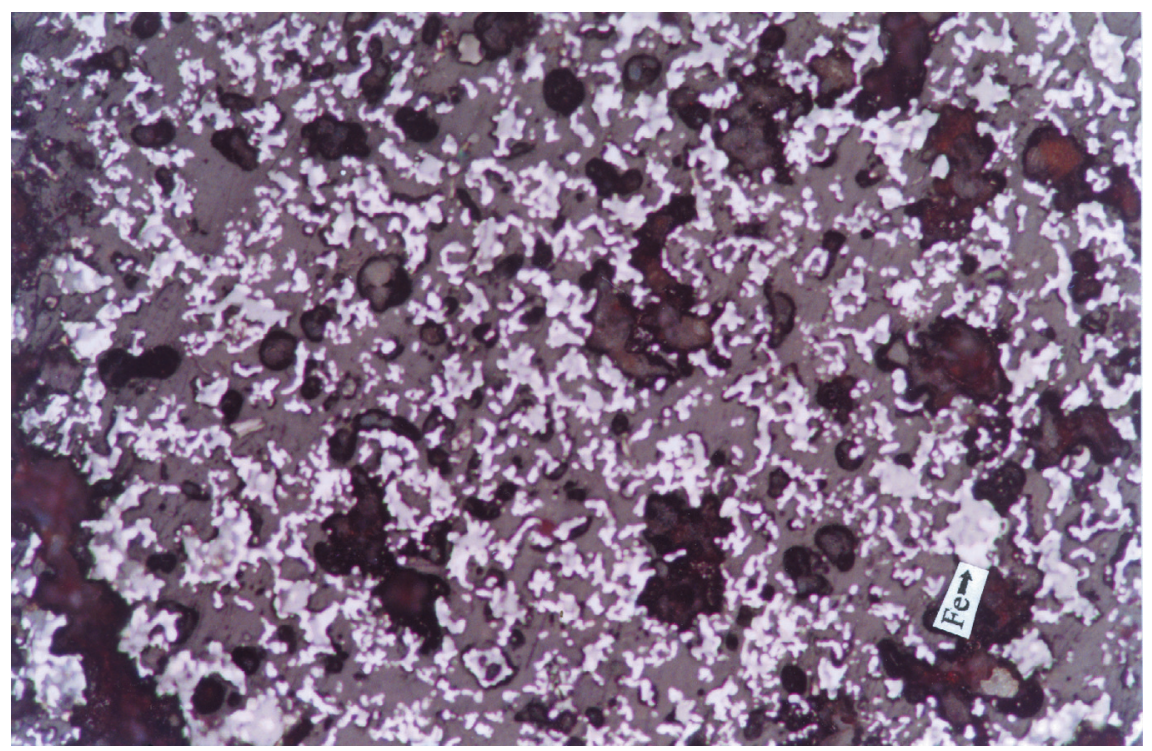

Foto 2 - Teste 10 realizado com coque (15,3\% em peso) à temperatura de $1120^{\circ} \mathrm{C}$, tempo de 30 minutos e com $43,3 \%$ de metalização (centro $100 x$ ). 
A Foto 5 é muito interessante, pois pode ser observado, com clareza, que a parte externa da borda, além de estar bem oxidada, tem uma característica dendrítica, sinalizando para uma fusão de grãos. Outro detalhe importante é a diferença acentuada entre a Foto 4 (parte central), que está bem metalizada, e a Foto 5 (borda), que se encontra muito oxidada.

É interessante também comparar esse conjunto de fotos, com a Foto 3 , que foi feita sob condições de testes semelhantes, exceto pelo tempo prolongado.

Na Foto 6, são mostradas as regiões de uma pelota bastante heterogênea. Pode- se ver regiões de alta metalização e regiões de baixa metalização, onde a presença dos óxidos é predominante. É importante salientar que o grau de metalização desse teste foi de $82,3 \%$, ou seja, a região não metalizada se concentrou em um lado da pelota, que é justamente o lado que se encontra próximo à parede do recipiente, o que, de certa forma, impermeabiliza a superfície .

Pode ser observado que a distribuição da fase metálica na pelota é bastante homogênea e a não homogeneidade de algumas pelotas se deveu à sua posição dentro do recipiente. Geralmente o lado mais metalizado é o que estava mais exposto à atmosfera do ambiente dentro do forno.

\section{Conclusões}

Através da microestrutura, observa-se, com clareza, que tempo de exposição prolongado a altas temperaturas ocasiona uma reoxidação do metal. De maneira geral, a distribuição de metal na pelota é bastante homogênea e a não homogeneidade de algumas pelotas se deveu à sua posição dentro do recipiente. Observou-se que o lado mais metalizado foi o que ficou mais exposto à atmosfera do ambiente.

\section{Agradecimentos}

Esse trabalho foi possível graças

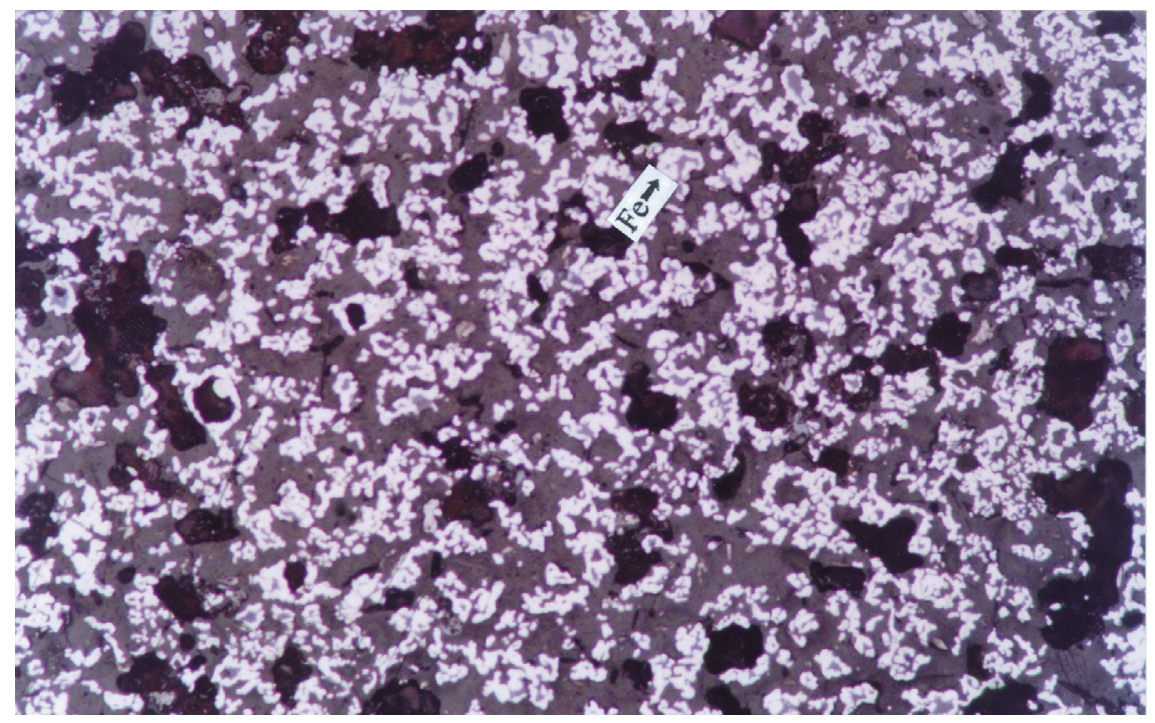

Foto 3 - Teste 11 realizado com coque (18,3\% em peso) à temperatura de $1120^{\circ} \mathrm{C}$, tempo de 30 minutos e com $89,3 \%$ de metalização (centro $100 x$ ).

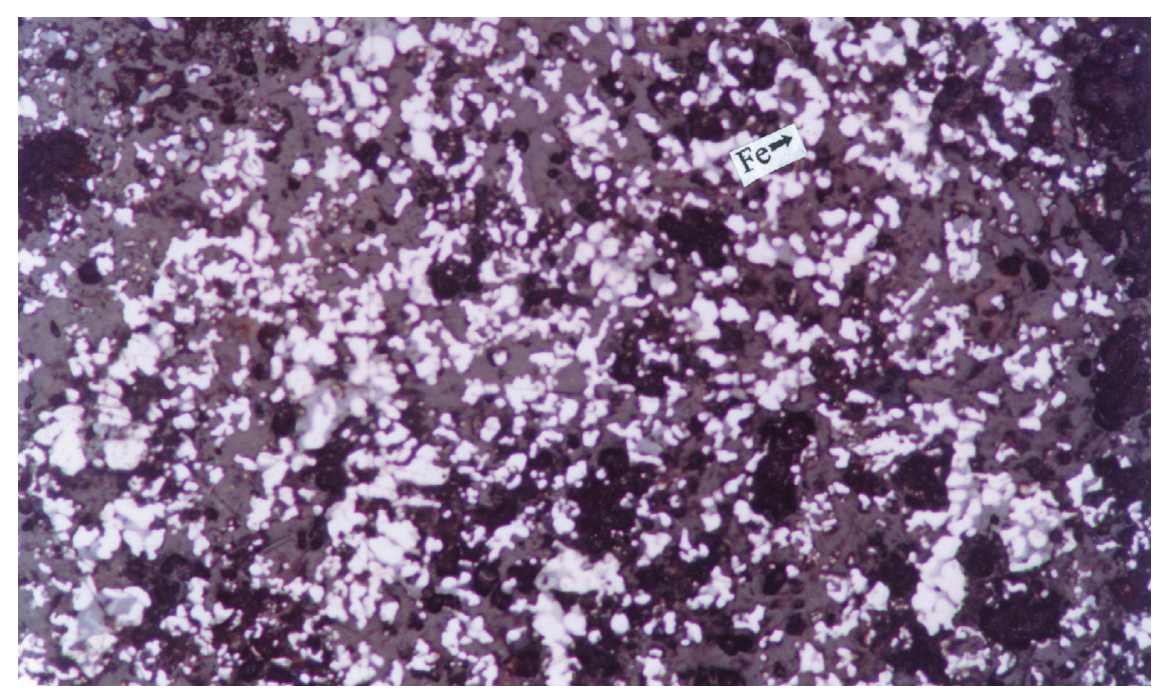

Foto 4 - Teste 33 realizado com coque (18,3\% em peso) à temperatura de $1120^{\circ} \mathrm{C}$, tempo de 90 minutos e com 45,1\% de metalização (centro 100x).

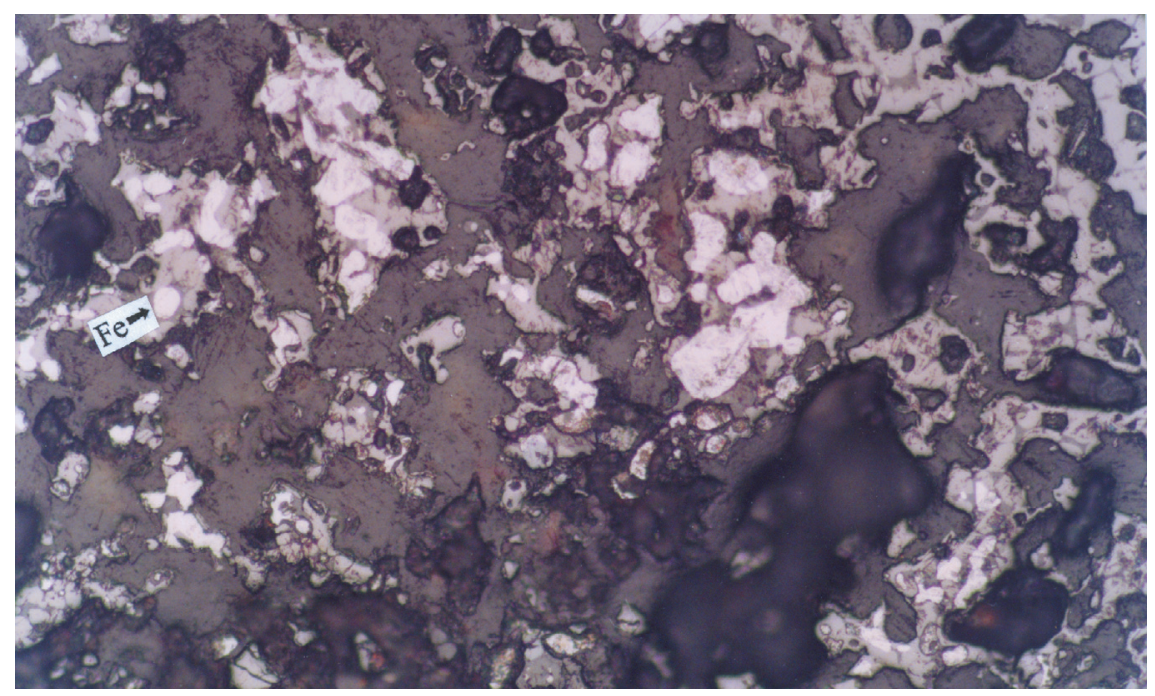

Foto 5 - Teste 33 realizado com coque (18,3\% em peso) à temperatura de $1120^{\circ} \mathrm{C}$, tempo de 90 minutos e com 45,1\% de metalização (borda 200x). 


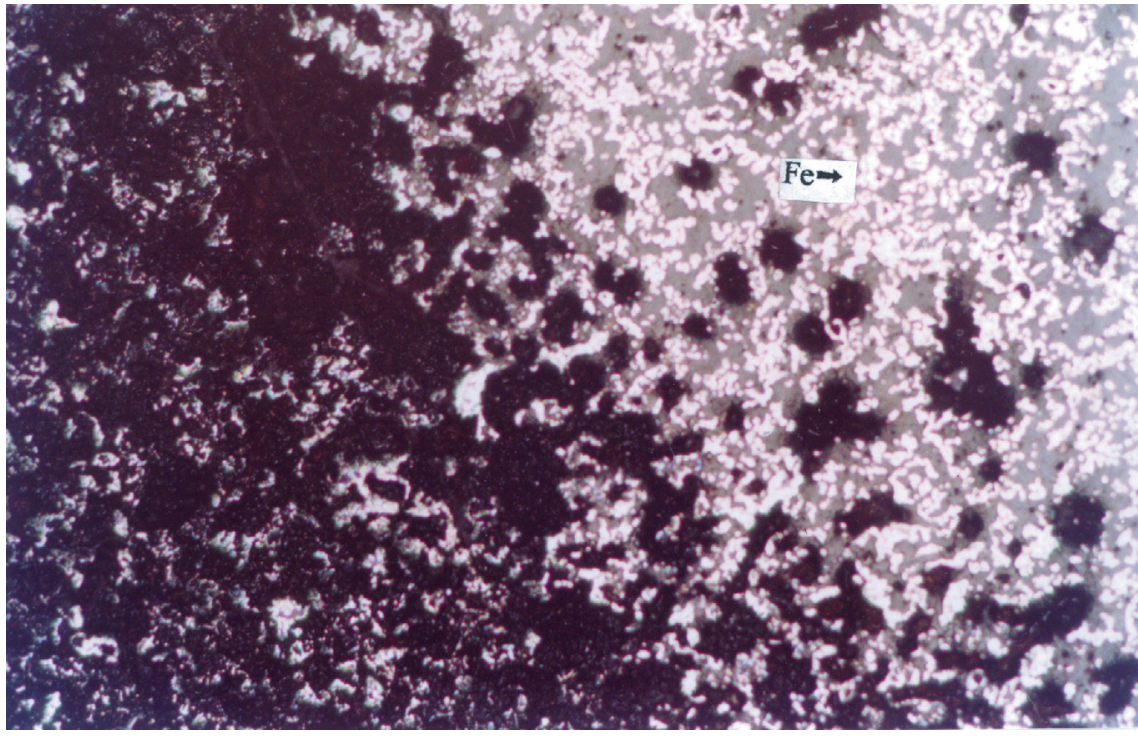

Foto 6 - Teste $D$ realizado com coque $\left(18,3 \%\right.$ em peso) à temperatura de $1180^{\circ} \mathrm{C}$, tempo de 20 minutos e com $83,2 \%$ de metalização (centro 50x).

ao financiamento de projeto apoiado pela Fundação de Amparo à Pesquisa do Estado de Minas Gerais - FAPEMIG.

\section{Referências \\ bibliográficas}

1 - Battele, Report - Reduction process outside the blast furnace and their effect on future iron steel production in the world, 1973.

2- BROSH, C. D. Fusão de pelotas auto redutoras em forno cubilô. In: CONGRESSO ANUAL DA ABM, 19. São Paulo: 1964.

3 - ASTIER, J. E. Tendências tecnológicas na produção de aço - Metalurgia e Materiais, Março, 1995

4- VÍVIAN, R. V., DIAS JUNIOR,O. S. Estado da arte da tecnologia de redução de minério de ferro e suas inovações, Metalurgia $\boldsymbol{e}$ Materiais, ABM, v. 48, 1992.

5 - PIZA, F.A.T. Redução direta de minério de ferro a partir de misturas minério-carvão. In: CONGRESSO ANUAL DA ABM, 3. São Paulo: 1974.
6- CAVANAGH, P. E. Manufacture of sponge iron process. J. Canad. Ceram. Soc. 1950

7- BOGDANDY, L., VON DAN ENGELL, H. J. The reduction of iron ore, Overla Stahleisen. Dusseldorf, 1971

8- MARTINS, J., GRESTA, E. R. Levantamento de dados preliminares visando à produção de ferro-esponja a partir de pelota crua composta de minério de ferro e carvão vegetal. In: CONGRESSO ANUAL DA ABM, 28. 1983.

9- STEFFEN, R. The corex process: first results in hot- metal making, Metallurgical Plant Technology, 1990.

10- LEINSKI, J. A., GRISCOM F. M. The midrex fastmet process, Midrex Direct Reduction Corporation, 1992.

11 - BAUER, K., HUTTE, D. Recycling of iron and steelworks wastes using the Inmetco direct reduction process, Metallurgical Plant and Tecnology International, v. 4, 1990.

12- MARTINS, J., GRESTA, E. R. Preliminary datas on the process of production of sponge iron from pellets made out of mixture of iron ore and charcoal. INT. SYMP. ON BENEFICIATION AND AGGLOMERATION, Bhubaneswar, India, 1986.

13- MARTINS, J., LIMA, N.P., SILVA, L.F. Efeito das variáveis: tipos de agente redutor, quantidade de agente redutor, taxa de aquecimento e tempo à temperatura máxima sobre o grau de metalização de pelotas. In: CONGRESSO ANUAL DA ABM, 54. São Paulo: Setembro, 1999.

14- SANTOS ASSIS, P., SAMPAIO R. S. Novos processos de produção de ferro primário Belo Horizonte: ABM, 1995

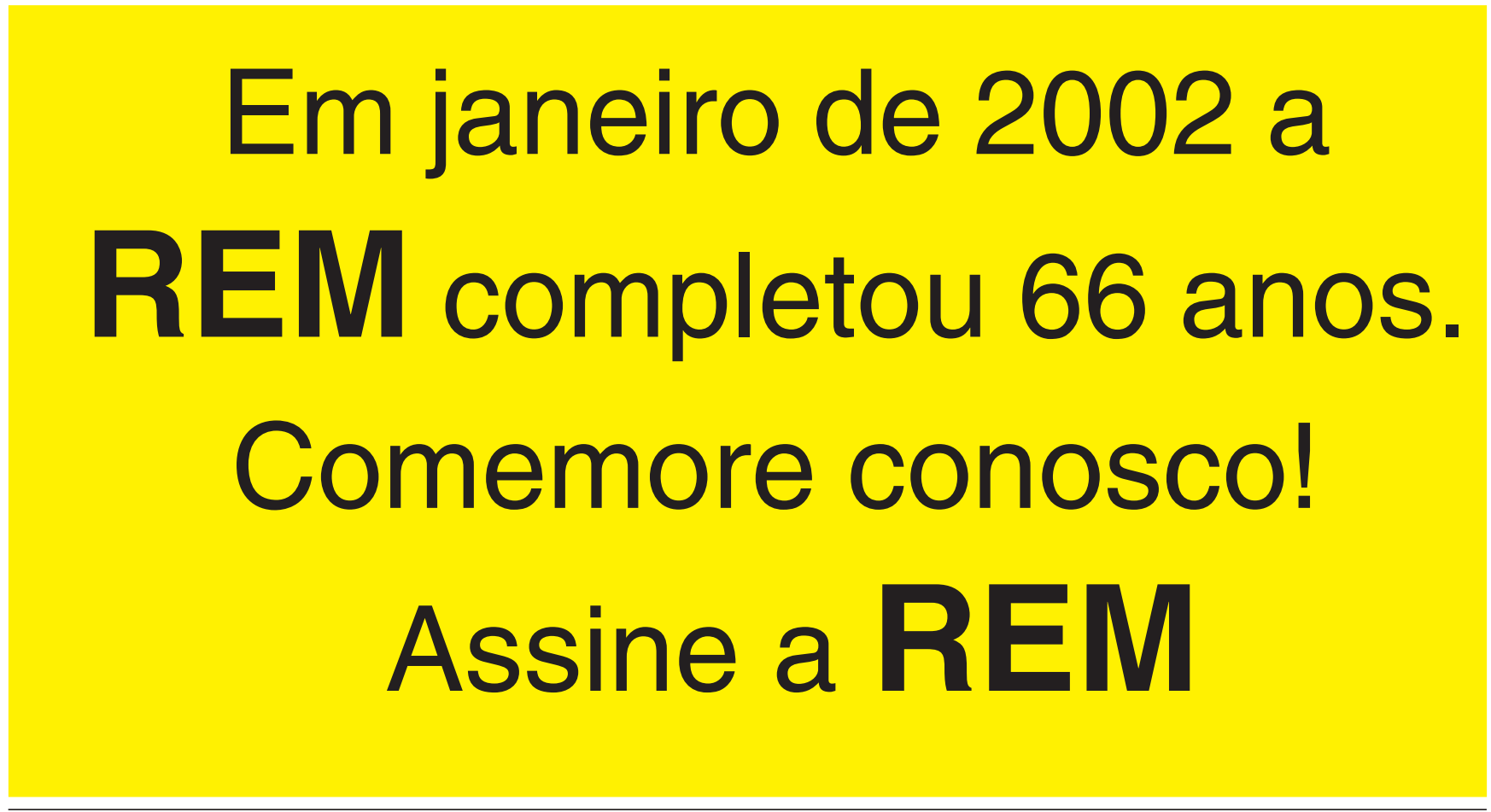

